\title{
Multisample Preparation Methods for the Solvent-free MALDI-MS Analysis of Synthetic Polymers
}

\author{
Sarah Trimpin \\ University, Portland, Oregon, USA \\ Charles N. McEwen \\ DuPont Corporate Center for Analytical Sciences, Wilmington, Delaware, USA
}

Center for Research on Occupational and Environmental Toxicology (CROET), Oregon Health and Science

\begin{abstract}
A limitation of any current approach using solvent-free MALDI mass spectrometry is that only one sample at a time can be prepared and transferred to the MALDI-plate. For this reason, multiple-sample preparation approaches for solvent-free MALDI MS analysis of synthetic polymers were developed that are simple and practical. One approach multiplexed sample preparation by simultaneously preparing multiple samples. With this approach, as many as 384 samples could be prepared by addition of analyte, matrix, salt, and 1-mm metal beads to each well of a 384-well disposable bacti plate, capping the plate with the lid and homogenizing all samples simultaneously using a common laboratory vortex device. Besides the time savings achieved by a single vortex step for multiple samples, an additional advantage of this method relative to previously reported solvent-free preparation methods is that the mixing volume per sample is reduced, which allows a reduction in the amount of analyte required. This method, however, still requires the transfer of each homogenized sample to the MALDI plate for subsequent analysis. Here we report a novel approach that combines multiple simultaneous solvent-free sample preparation with automatic sample transfer to the MALDI target plate. This approach reduces the possibility of cross-contamination, the amount of sample and matrix consumed for an analysis, and the time required for preparation of multiple samples. These methods were shown to provide high-quality mass spectra for various synthetic polymer standards with $M_{n}$ values to $10 \mathrm{kDa}$. The methods are efficient in that small sample amounts are required, the sample/salt/matrix ratio is not critical, and the time necessary to achieve sufficient homogenization of multiple samples is less than $5 \mathrm{~min}$. (J Am Soc Mass Spectrom 2007, 18, 377-381) (C) 2007 American Society for Mass Spectrometry
\end{abstract}

$\mathrm{N}$ umerous experiments have revealed analytical advances in the solvent-free analysis of different types of analytes including synthetic polymers. A significant advantage of solventfree MALDI analysis is that the method is capable of characterizing insoluble compounds such as polyfluorene [1] and is consequently routinely used for the characterization of large polycyclic aromatic hydrocarbons [2]. A recent study based on the ball-mill homogenization/loose powder transfer method revealed that solvent-free MALDI-MS simplifies the measurement and improves the result qualitatively and quantitatively [3]. In general, the solvent-free method gives more homogeneous analyte/matrix

Published online November 20, 2006

Address reprint requests to Dr. Sarah Trimpin, Oregon Health and Science University, CROET, 3181 SW Sam Jackson Park Rd., Portland, OR 97239.

E-mail: sarah.trimpin@oregonstate.edu mixtures and higher shot-to-shot and sample-tosample reproducibility than does the solvent-based method $[3,4]$. Consequently, less laser power has to be applied [3,4], which yields milder MALDI conditions and thus less fragmentation [1,3]. This method, for the same reasons, also reduces background signals [3] and improves the resolution of the analyte signals $[3,4]$.

For synthetic polymer analyses, three general methods exist for homogenization. These are grinding by mortar and pestle [4, 5], ball-mill [1-4], and vortexing [6]. Analyte, matrix, and salt are added to a vessel and, in the case of the ball-mill or vortex methods, suitably sized balls are used to assist in the homogenization process. For polymers having $M_{n} \leq 10 \mathrm{kDa}$ ball-mill, vortex, and mortar-and-pestle approaches appear to work equally well $[4,6]$. Two general methods exist for the dry transfer of the homogenized powder from the vessel to the MALDI plate. In one method a pressed 
pellet is affixed to the plate with double-sided adhesive tape [5] and in another a loose powder [1-4] is transferred by the use of a small spatula, gently pressing it to the plate and producing a thin film.

Solvent-free MALDI simplifies the sample preparation by removing the influence of solvent and provides, in cases where the solvent is problematic, considerable time savings. However, for samples in which the solvent is not an issue, the solvent-free method is generally less efficient. Workflow and clean-up issues related to solvent-free MALDI analysis were considered serious handicaps for high-throughput applications. These reservations are partially overcome by the vortex [6] and mini-ball-mill (MBM) methods [7-9]. An on-target grinding method, initially demonstrated with a polystyrene (PS) 5050 standard [6], was accomplished by simply adding, without weighing, the matrix, polymer, and salt together on the MALDI plate and homogenizing them with a metal spatula [10]. Although the acquired mass spectra were of poorer quality than with the vortex method, this work demonstrated the feasibility of on-target sample preparation.

In the work described herein, the single vortex method [6] was multiplexed by using an appropriate sample holder. This multisample method was then extended by developing a strategy for simultaneous homogenization with parallel transfer of the matrix/ analyte/salt mixture directly onto the MALDI plate. The method uses common laboratory materials and a simple vortex device. Inclusion of simultaneous sample transfer with multiplex homogenization reduces the effort and time required to prepare multiple samples by the solvent-free approach and makes this approach competitive with solvent-based methods for low molecular weight (MW) polymer analyses.

\section{Experimental}

\section{Materials}

2,5-Dihydroxybenzoic acid (DHB), dithranol, all-transretinoic acid, and sodium trifluoroacetate were obtained from Aldrich (Milwaukee, WI) and used without further purification. Narrow polydispersity (PD) poly(ethylene glycol) (PEG) standards with $M_{p}=600,1470$, 4250 , and 7100 and poly(methyl methacrylate) (PMMA) with $M_{p}=1830,2990,3800,5270,6950,10,300,15,100$, and 21,600 were obtained from Polymer Laboratories (Church Stetton, UK). A sample was prepared to mimic a wide PD polymer by mixing equal weights of these PMMA standards. Polystyrene narrow distribution standards with $M_{p}=5050,7200,14,000$, and 24,200 were obtained from American Polymer Standards Corp. (Mentor, $\mathrm{OH}$ ).

\section{Mass Spectrometer}

The mass measurements were performed on a PerSeptives Biosystems (Framington, MA) Voyager-DE STR
MALDI instrument using a nitrogen laser and a laser power level slightly above threshold for analyte signal.

\section{Solvent-free MALDI Multisample Preparation}

Two vortex methods were evaluated for effectiveness in homogenizing solvent-free MALDI samples.

Method 1a. Microcentrifuge PCR tubes $(200 \mu \mathrm{L})$ (Molecular Bioproducts, San Diego, CA) were placed in the 96 slots of a $20 \mu \mathrm{L}$ Eppendorf pipette holder. Various polymers were placed in each tube in an amount that approximated $0.3 \mathrm{mg}$ for the $200 \mu \mathrm{L}$ tubes. Matrix (DHB or dithranol, about 1-5 mg) along with a few crystals of table salt $(\mathrm{NaCl})$ were added to the tubes followed by addition of two to five stainless steel balls $(1.2 \mathrm{~mm}$, BioSpec Products, Bartlesville, OK). The vials were capped and held in place with a flat plate and the entire device was taped to hold it together during the 3- to 5-min vortex procedure.

Method 1b. An alternate approach uses a 96- or 384well bacti plate (Nunc, Roskilde, Denmark). In this approach, about $1 \mathrm{~g}$ of matrix and $0.1 \mathrm{~g}$ of a sodium acetate were premixed and vortexed for 1 min using the BB/scintillation vial approach [6]. Typically, about 1 $\mathrm{mg}$ of premixed matrix/salt, several $1.2 \mathrm{~mm}$ stainless steel balls, and analyte $(<0.1 \mathrm{mg})$ are added to each well and the plate covered with parafilm (American National Can, Greenwich, CT) followed by its cover plate. As above, the entire device, held together with tape, is placed on a vortex device for up to $5 \mathrm{~min}$. In both methods, the samples were transferred to the MALDI plate using the wooden end of a cotton tip applicator (Qtip; Puritan, Worthing, UK). Loose powder was removed in a hood with a gas stream from a container of difluoroethane or with a clean nitrogen gas stream.

\section{Parallel On-target Homogenization of Multiple Samples Using a TissueLyser}

Two methods were explored for simultaneous homogenization and sample transfer to the MALDI plate.

Method 2, TissueLyser method. Samples were prepared as above (method 1a) using $2 \mathrm{~mL}$ microcentrifuge tubes but with the premixed matrix/salt (10:1) [3]; the amount of metal beads corresponded to roughly half the volume of the matrix. Eight uncapped tubes were placed in each of the two TissueLyser (Qiagen, Valencia, CA) adapter sets. A MALDI plate was used to cap each set of eight tubes and fixed in place using tape before closing the adapter set lid. The sets were shaken for $2 \mathrm{~min}$. and the MALDI plates removed from the set (Supplementary Material data, which can be found in the electronic version of this article, Figure 1A). Loose matrix/analyte powder was dusted off (Supplementary Material data, Figure 1B) using a stream of difluoroethane gas. 


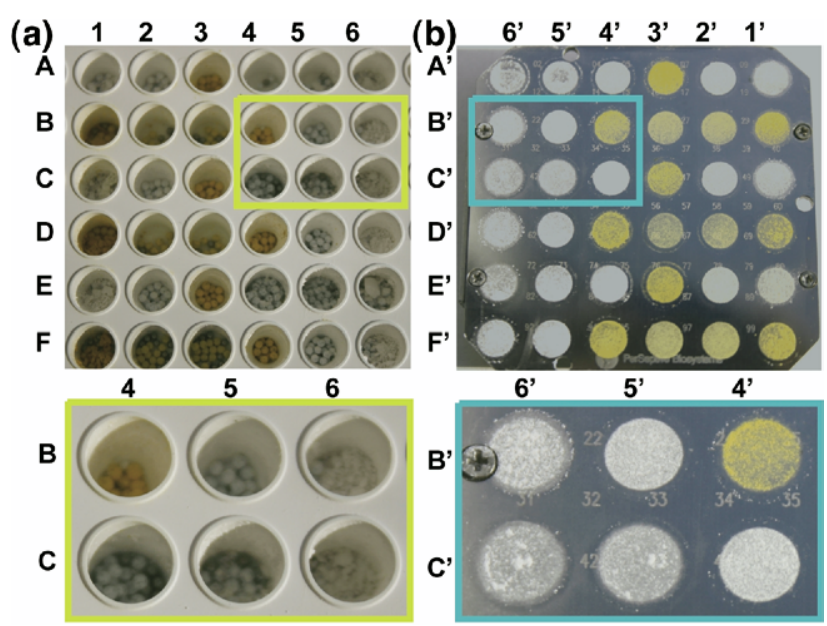

Figure 1. Photographs of the loaded bacti plate (a) and the mirror image MALDI plate of the homogenized/transferred MALDI samples (b) using the vortex device for $5 \mathrm{~min}$ as well as their respective enlargements (bottom). Wells A3, B1-4, C3, D1-4, E3 and F1-4 used dithranol as matrix and all other wells used 1,5 dihydroxybenzoic acid as matrix.

Method 3, Vortex method. A MALDI plate exactly fits twenty-five $200 \mu \mathrm{L}$ Eppendorf tubes (method 3a), or 36 wells of the Nunc bacti 96-well plate (122 wells of the 384-well plate) (method 3b). Samples (25) were prepared as in Method 1 (Supplementary Material data, Figure 2). In the case of the 36 samples after 2 to $5 \mathrm{~min}$. on the vortexer, the cover plate and parafilm were removed and a MALDI plate was used to cap the wells (Figure 1a). Tape was used to hold the MALDI plate firmly against the bacti plate and the entire device was vortexed for an additional $1 \mathrm{~min}$. to transfer the homogenized sample/matrix. Alternatively, the bacti-plate with MALDI plate attached can be vortexed for the entire time (Figure 1b) with $5 \mathrm{~min}$. homogenization/ transfer giving better results than $2 \mathrm{~min}$. (Supplementary Material data, Figure 2). Again, loose powder was removed from the target plate (Figure 1b) before the plate was placed in the mass spectrometer.

\section{Results and Discussion}

The simplicity and widespread use of vortex devices seemed appropriate for extension of the two-sample MBM [7] set to 96 or 384 samples simultaneously (method 1). This approach is, in principle, usable for $n$-samples. Thus, the sample numbers and volume that can be prepared simultaneously is limited by the multisample holder and the ability to achieve vigorous shaking of all cavities in the device. The smaller tubes in the multiple vortex approach required smaller beads, which subsequently required longer times on the vortexer than the single-sample vortex method [6], which uses two BBs (4.5 mm steel balls, about $1 \mathrm{~min}$. homogenization). The results could also be improved by using a larger number of smaller beads or by including a single larger metal ball with the smaller beads, provid- ing the wells were sufficiently large as in the 96-well bacti plate. For the latter, excellent results were obtained for PS 5050 and 7200 after vortexing for just 3 $\mathrm{min}$. Overall, the time savings corresponds to just over $1.5 \mathrm{~h}$ considering 96 samples and using 5 -min vortex homogenization as compared to the single-sample vortex approach previously described [6].

Because analyte/matrix transfer to the MALDI target plate is a labor-intensive process, a feasibility study was undertaken to determine whether the above approach could be extended to include simultaneous sample transfer. A second set of experiments (method 2) used a TissueLyser to simultaneously prepare and transfer 16 polymer standards with different molecular weights and polydispersities onto two MALDI plates (Supplementary Material data Figure 1; 2 min. homogenization/transfer). The TissueLyser gives more vigorous shaking than the laboratory vortex devices. As can be seen in Figure 2, after 2 min on the TissueLyser, mass spectra were obtained on narrow polydispersity PEG and PMMA as well as on an equal weight mixture of PMMA narrow PD standards designed to mimic a wide PD sample. However, oligomers in the PMMA wide PD

(a)

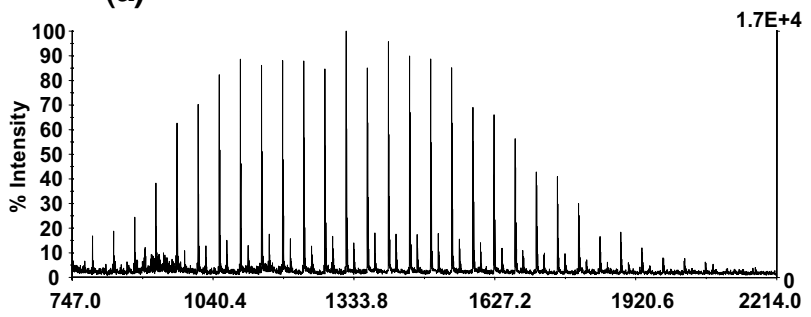

(b)

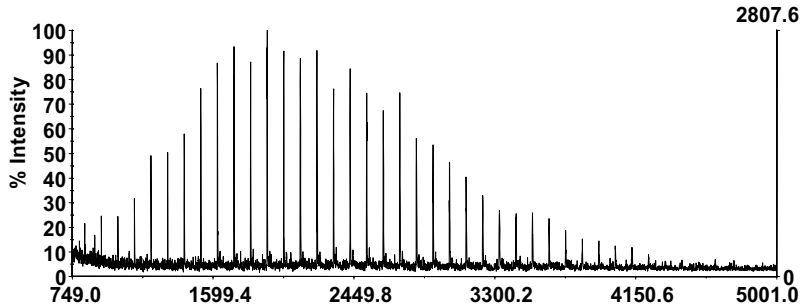

(c)

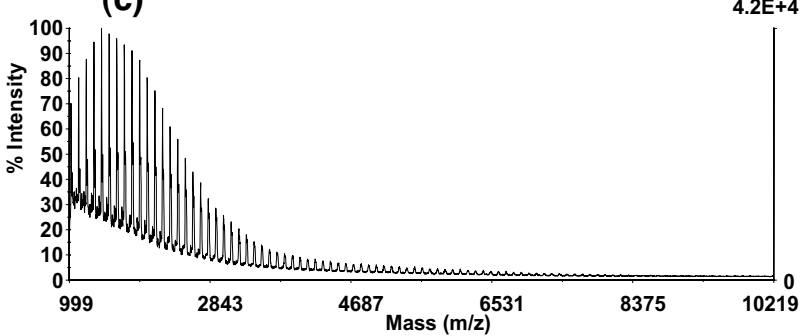

Figure 2. On-target homogenization/transfer solvent-free MALDI mass spectra using the TissueLyser approach for (a) PEG 1470; (b) PMMA 1830; (c) A mixture of poly(methylmethacrylate) (PMMA) narrow distribution standards including PMMA 1830, $2400,2990,3800,5270,6950$, and 10,300 in an equiweight ratio designed to mimic a broad polydisperse polymer. The mass measurements were obtained 5 days after the sample preparation. 
mixture did not show ions much above $10 \mathrm{kDa}$, a result not unexpected for a wide PD polymer. The method is fast and cross-contamination free (a tightly closed system prevents tube-to-tube spillover; Supplementary Material data Figure 1A).

Although the TissueLyser approach was successful, the method would be more useful if the homogenization/transfer should also be successful using a larger number of samples per MALDI plate and using a commonly available vortex device (method 3 ). The bacti plate used in method 1 was used for this experiment and allowed 36 (96-well) or 122 (384-well) samples to be prepared simultaneously on a MALDI plate. The prepared MALDI plate is shown in Figure 1 (for 36 samples) after $5 \mathrm{~min}$. on a vortex device and after dusting off the excess powder. Extending the vortex time from $1 \mathrm{~min}$. (Supplementary Material data, Figure 2.I), to $5 \mathrm{~min}$. (Supplementary Material data, Figure 2.II) produced a finer and, presumably, a more homogeneous coverage, and improved the signal-to-noise ratio and reproducibility for analyte ions. Thus, the vortex period is longer in this method than, for example, either the single-sample vortex/BB method or the TissueLyser multisample method. The use of a single larger metal ball (BB) along with a few smaller beads gave excellent spectra and reproducibility for PS 5050 and 7200 samples with $3 \mathrm{~min}$. total vortex time.

The shot-to-shot and sample-to-sample reproducibility for both analyte and matrix were good, as exemplified for the results obtained for PS 7200 (Figure 3, IA and IB). Cross-contamination was not observed. The fact that PEG, PS, and PMMA all gave good mass spectra for $M_{p}$ values up to and including PMMA 10,300 (Figure 3, II.A) demonstrates that this method is flexible and compatible with different samples and molecular weights. For the latter, the results are also comparable with the solvent-based MALDI results (Figure 3, II.B), which were obtained by dissolving the solvent-free sample with THF directly on target.

With the current procedures we are able to obtain mass spectra on PMMA $21.6 \mathrm{kDa}$ by using five to ten $1.2-\mathrm{mm}$ beads and $10 \mathrm{~min}$. vortex time (method $3 \mathrm{a}$, Supplemental Figure 3); however, these results are not reproduced from sample to sample. Apparently as the MW of the polymer increases the ability to homogenize the analyte and matrix becomes more daunting. The vortex device may be limited in its ability to homogenize higher MW polymers because the mini-ball-mill method gave good quality and reproducible spectra even for the highest MW polymer (PS 24,200) we attempted.

Method 3 (96-well bacti plate) was used to prepare 18 randomly selected proprietary polymer samples submitted for analysis over the past 12 months. Although randomly chosen, all samples had previously been successfully analyzed using either solvent-free or solvent-based MALDI and all had $M_{n}$ values $<10 \mathrm{kDa}$. Each sample was prepared using either DHB and dithranol as matrix and appropriate

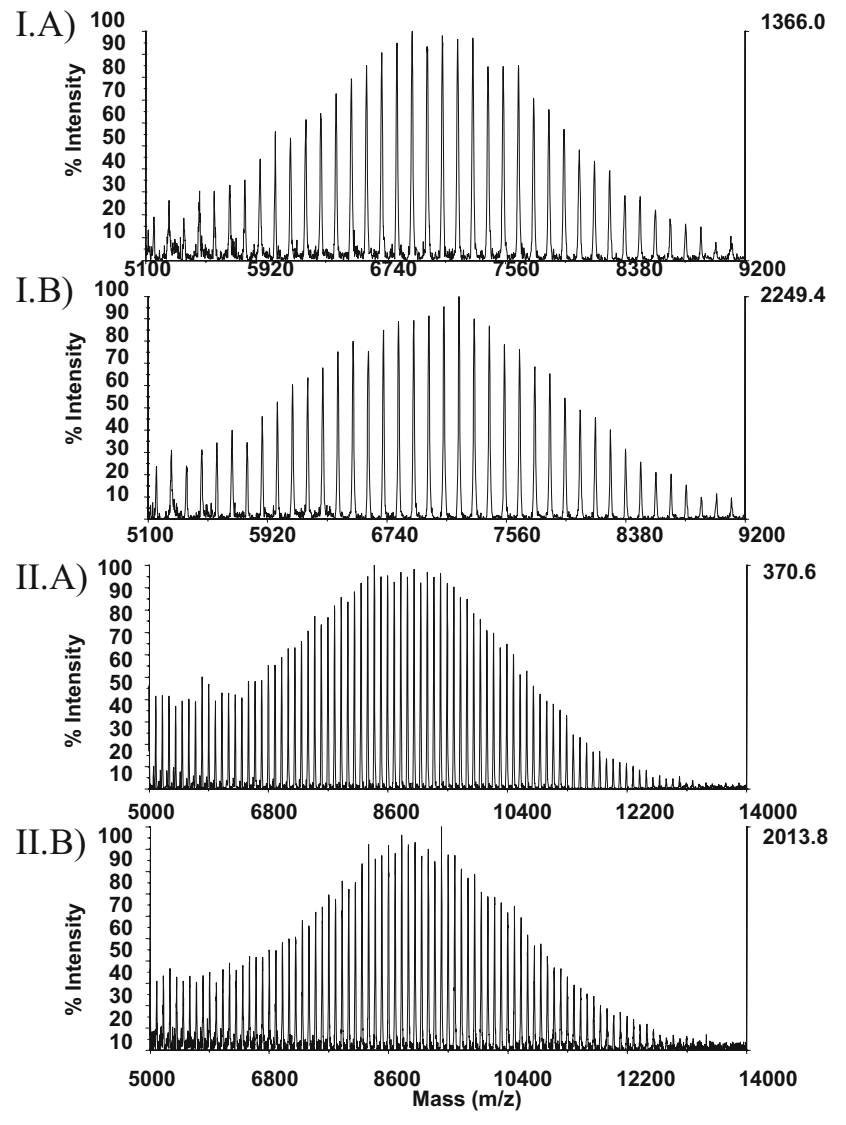

Figure 3. Mass spectra of PS 7200 (IA and IB) investigating spot-to-spot reproducibility and PMMA 10,300 (IIA) (Figure 2, in Supplementary Material data), using on-target homogenization/ transfer solvent-free MALDI with the vortex device compared to a solvent-based approach (IIB) by adding THF to the PMMA/ matrix/salt mixture used to obtain spectra IIA.

salt. Satisfactory results were obtained for all 18 samples using either matrix.

Method 3, which uses simultaneous homogenization and transfer, is a considerable simplification of the solvent-free MALDI process. Even in cases where there are few samples, this multiplex approach reduces the task of testing various matrix materials, or salt combinations to achieve the most satisfactory results. We are currently exploring methods to further simplify the procedures used to load the matrix, salt, and analyte in each well. Using the 36-sample bacti plate approach of method 3, the time to load the wells and homogenize the analyte/matrix with simultaneous transfer to the MALDI target currently is $<1 \mathrm{~min} /$ sample.

\section{Conclusions}

A multisample approach for simultaneous direct on-target MALDI sample preparation has been developed that standardizes and automates the labor-intensive homogenization and sample transfer steps in preparing a MALDIplate. Because the solvent-free method provides good shot-to-shot reproducibility, automatic sample acquisition is expected to be reliable. This implies that a multisample 
approach could make high-throughput solvent-free MALDI-MS a reality. In addition to the time and labor savings, the amount of analyte needed for a successful analysis has been reduced, suggesting that solvent-free MALDI MS may be applicable for analyses where sample amounts are limited.

\section{Acknowledgments}

This work was supported by US PHS NIH-NIEHS grants ES 10338 and ES 011384, and the Oregon Workers Benefit Fund. The MS Core of the EHSC and the NIH/NIEHS grant ES00040 were also used in support of this work. The authors thank Prof. M. L. Deinzer (vortexbased studies), OSU, Corvallis, OR and Prof. P. S. Spencer (TissueLyser-based studies), CROET, Portland, OR, for providing the opportunity to use their laboratory for sample preparation and Courtney Runyan for technical assistance in using the TissueLyser.

\section{References}

1. Trimpin, S.; Grimsdale, A. C.; Räder, H. J.; Müllen, K. Characterization of an Insoluble Poly(9,9-diphenyl-2,7-fluorene) by Solvent-Free Sample
Preparation for MALDI-TOF Mass Spectrometry. Anal. Chem. 2002, 74, 3777-3782.

2. Simpson, C. D.; Mattersteig, G.; Martin, K.; Gherghel, L.; Bauer, R. E.; Räder, H. J.; Müllen, K. Nanosized molecular propellers by cyclodehydrogenation of polyphenylene dendrimers. J. Amer. Chem. Soc. 2004, 126(10), 3139-3147.

3. Trimpin, S.; Keune, S.; Räder, H. J.; Müllen, K. Solvent-free MALDI-MS: Developmental Improvements in the Reliability and the Potential of MALDI Analysis of Synthetic Polymers and Giant Organic Molecules. J. Am. Soc. Mass Spectrom. 2006, 17, 661-671.

4. Trimpin, S.; Rouhanipour, A.; Az, R.; Räder, H. J.; Müllen, K. New Aspects in Matrix Assisted Laser Desorption/Ionization Time-of-Flight Mass Spectrometry: A Universal Solvent-free Sample Preparation. Rapid Commun. Mass Spectrom. 2001, 15, 1364-1373.

5. Skelton, R.; Dubois, F.; Zenobi, R. A MALDI. Sample Preparation Method Suitable for Insoluble Polymers. Anal. Chem. 2000, 72, 17071710.

6. Hanton, S. D.; Parees, D. M. Extending the Solvent-free MALDI Sample Preparation Method. J. Am. Soc. Mass Spectrom. 2005, 16, 90-93.

7. Trimpin, S.; Deinzer, M. L. Solvent-free MALDI-MS for the Analysis of Biological Samples via a Mini-Ball Mill Approach. J. Am. Soc. Mass Spectrom. 2005, 16, 542-547.

8. Trimpin, S.; Deinzer, M. L. Solvent-free MALDI-MS for the Analysis of a Membrane Protein via the Mini-Ball Mill Approach: a Case Study of Bacteriorhodopsin. Anal. Chem., in press.

9. Trimpin, S.; Deinzer, M. L. Solvent-free Mass Spectrometry for Hydrophobic Peptide Sequence Analysis and Protein Conformation Studies. Biotechniques 2005, 39, 799-805.

10. Private communication from Dr. Scott Hanton (Air Products). 